\title{
Fractional diffusion modeling of ion channel gating
}

\author{
Igor Goychuk: and Peter Hänggi \\ Institute of Physics, University of Augsburg, Universitätsstr. 1, D-86135 Augsburg, Germany
}

(Dated: August 6, 2021)

\begin{abstract}
An anomalous diffusion model for ion channel gating is put forward. This scheme is able to describe non-exponential, power-law like distributions of residence time intervals in several types of ion channels. Our method presents a generalization of the discrete diffusion model by Millhauser, Salpeter and Oswald [Proc. Natl. Acad. Sci. USA 85, 1503 (1988)] to the case of a continuous, anomalous slow conformational diffusion. The corresponding generalization is derived from a continuous time random walk composed of nearest neighbor jumps which in the scaling limit results in a fractional diffusion equation. The studied model contains three parameters only: the mean residence time, a characteristic time of conformational diffusion, and the index of subdiffusion. A tractable analytical expression for the characteristic function of the residence time distribution is obtained. In the limiting case of normal diffusion, our prior findings [Proc. Natl. Acad. Sci. USA 99, 3552 (2002)] are reproduced. Depending on the chosen parameters, the fractional diffusion model exhibits a very rich behavior of the residence time distribution with different characteristic timeregimes. Moreover, the corresponding autocorrelation function of conductance fluctuations displays nontrivial features. Our theoretical model is in good agreement with experimental data for large conductance potassium ion channels.
\end{abstract}

PACS numbers: 05.40.-a, 87.10.+e, 87.15.He, 87.16.Uv

\section{INTRODUCTION}

Ion channels are complex membrane proteins which provide ion-conducting, nanoscale pores in the biological membranes [1]. These proteins undergo spontaneous conformational dynamics resulting in stochastic intermittent events of opening and closing the pore - the so-called gating dynamics. It can be described by following kinetic scheme:

$$
C \underset{k_{c}}{\stackrel{k_{o}}{\rightleftarrows}} O
$$

As it stands, this scheme describes Markovian stochastic transitions between the closed state (C) and the state open (O) of an ion channel which can fully be characterized by the opening rate, $k_{o}$, and the closing rate, $k_{c}$. From a trajectory description of the observed two-state gating process, these transitions can be characterized by the residence time distributions (RTDs) of open and closed time intervals, $\psi_{o}(\tau)=k_{c} \exp \left(-k_{c} \tau\right)$ and $\psi_{c}(\tau)=k_{o} \exp \left(-k_{o} \tau\right)$, respectively.

The invention of patch clamp technique [2] marked the beginning of a new era: detailed experimental studies of the statistics of such stochastic trajectory realizations have been rendered possible. These experimental investigations, however, also reveal the fact that the distributions of the residence time intervals are typically not exponential. This in turn implies that the corresponding observed two-state dynamics of current fluctuations is not Markovian. Any such non-exponential distribution can however approximately be fitted by a sum of (sometimes many) exponentials, e.g.

$$
\psi_{c}(t)=\sum_{i=1}^{N} c_{i} \lambda_{i} \exp \left(-\lambda_{i} t\right),
$$

with weights $c_{i}$ obeying $\sum_{i=1}^{N} c_{i}=1$. The rationale behind this fitting procedure is the assumption that the corresponding state consists of $N$ discrete substates, separated by potential barriers. This method constitutes the working tool for the majority of molecular physiologists in interpreting their experimental data within a discrete Markovian scheme consisting of many (sub)states [3]. The addition of new states (or new configurational dimensions in the continuous case) is a well known formal method to unravel a low-dimensional non-Markovian stochastic dynamics 
via its embedding into a Markovian dynamics of higher dimensionality. The problem with such a methodology is, however, that the number of substates needed to fit the experimental data can depend on the experimental conditions. For example, the experimental gating dynamics of a Shaker potassium channel has been successfully described by a sequential 8-state Markovian scheme with 7 closed states for a fixed value of temperature about $T=20{ }^{\circ} \mathrm{C}$ [4]. However, to describe the experimental data over a small extended temperature regime between $10-20^{\circ} \mathrm{C}$ already necessitates to add three additional closed substates [5].

For several types of ion channels the RTDs can alternatively be fitted in terms of non-exponential distributions such as a stretched exponential $\psi(\tau) \propto-\frac{d}{d \tau} \exp \left[-(\nu \tau)^{\alpha}\right][\underline{6}]$, or by a power law $\psi(\tau) \propto \tau^{-\beta}$ [], [] with a few parameters only. The case of a power law coefficient near $\beta=3 / 2$ can be described with normal conformational diffusion over many degenerate substates 17, 9, 10, 11, 12]. Such diffusion models 17, 9, 10, 11, 12, 13, 14, 15, 16] present an alternative to the standard discrete state Markovian modeling which can be considered also as a complementary one 16]. It should be noted, however, that exponents $\beta$ different from a normal diffusion behavior have been detected experimentally as well [17, 18, 19]. Therefore, it is of prominent importance to generalize the normal diffusion model to the case of anomalous diffusion: this objective is at the heart of the following discussion.

\section{MODELING ANOMALOUS ION CHANNEL GATING}

Ion channels are protein complexes with an intrinsically hyperdimensional conformational space. Such macromolecular complexes can possess several macroconformations corresponding to different functional states of the ion channel. These macroconformations are, however, not static, but rather present dynamical structures featuring multiple conformational substates and undergoing a corresponding intraconformational dynamics which can be of the diffusion type, as it is well studied for one of the simplest proteins - myoglobin [20, 21, 22]. It particular, for myoglobin it has been shown experimentally that the diffusive motions become increasingly important above some critical temperature and can serve as lubricants for macroconformational changes, see e.g. in Ref. [22]. There is no convincing reason to believe that ion channels, which are structurally much more complex proteins, are very different from myoglobin in this respect. Therefore, the conformational diffusion should be present and can be important for the gating dynamics of ion channels as well. Diffusion models of ion channel gating put emphasis on this fundamental feature of protein dynamics. In the case of ion channels with one open and one closed macroconformations the whole conformational space can be divided into the two hyperdimensional subspaces corresponding to the open macroconformation and to the closed macroconformation, respectively. These subspaces present generally some complex manifolds consisting of domains of attraction in the conformational space which are separated by potential barriers. Such domains of spatial localization can be associated with discrete states in the discrete state diffusion modeling of the gating dynamics. They can possess but a complex intrinsic structure reflecting the intrinsic multidimensionality of the problem. For this reason, such states can be trapping states with a non-exponential distribution of the residence times spent in the corresponding domains of attraction. On the contrary, the Markovian assumption within a discrete state modeling involves the assumption on strictly exponential distribution of the residence times in such discrete states. This presents at best an approximation only. It is at the heart of modeling the gating dynamics with appropriate Markovian kinetic schemes. Moreover, some parts of conformational potential landscape can lack the presence of pronounced barriers and some domains of attraction can be flat providing valleys in the corresponding potential landscape. This provides an ideal starting point for a diffusion modeling of the gating dynamics.

The simplest diffusion model [7, 9] assumes the existence of such a one-dimensional valley wherein the diffusion is modeled by sequential transitions among a large number of discrete substates. Alternatively, a continuous diffusion modeling can be applied [10, 11, 14, 15]. Note also that multiple pathways into the open state can be accounted for within a one-dimensional reaction coordinate modeling by allowing for transitions with non-nearest neighbor jumps. Such complexity will not be addressed within our sequential diffusion model. At one edge of this valley the transition into the open state can occur via the single route 7, 9, 10]. Such a modeling is appropriate when the correlations between the subsequent closed and/or open residence time intervals are absent. The multiple transitions could also induce some correlation between the durations of time intervals. Such effects will be neglected in a zero order approximation (renewal assumption for the observed two-state conductance fluctuations). The existence of time-correlations between the durations of time intervals (non-renewal gating dynamics) would indicate the presence of more than one route between the manifolds of open and closed states (see, e.g., in Ref. [2], pp. 440-441). Such correlations can also be accounted for in the diffusion models [14, 15. The incorporation of such correlations is, however, beyond the scope of our present work which presents an anomalous diffusion generalization of the Markovian modeling used in Ref. [7, 9, 10, 11].

Let us start from a continuous time random walk (CTRW) 23, 24, 25, 26, 27] generalization of the discrete state diffusion model by Millhauser et al. 7], see Fig. 1. It is assumed that the manifold of closed substates consists of $\mathrm{N}$ states; namely, the states from $j=2$ to $j=N-1$ ("diffusion states") are identical and characterized by identical 


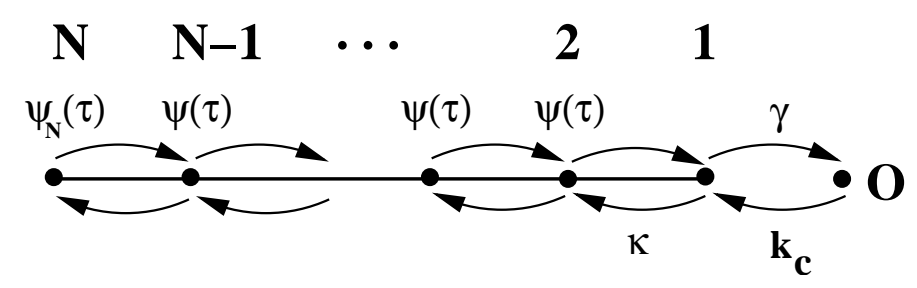

FIG. 1: Sketch of the CTRW generalization of the (discrete) diffusion model of ion channel gating.

residence time distributions $\psi_{j}(\tau)=\psi(\tau)$, i.e. the channel stays in the corresponding state $j$ for a random time interval $\tau$ distributed in accordance with $\psi(\tau)$, and performs at the end of every time interval a jump $j \rightarrow j \pm 1$ with probability $p_{j+1, j}=p_{j-1, j}=1 / 2$ either to the left, or to the right neighboring state, respectively. If the RTD is exponential, i.e. $\psi(\tau)=2 \kappa \exp (-2 \kappa \tau)$, then the standard Markovian rate description with rate $\kappa$ is recovered. The boundary state $j=N$ possesses a different RTD $\psi_{N}(\tau)$ which in the Markovian case reads $\psi_{N}(\tau)=\kappa \exp (-\kappa \tau)$ (transitions occur always with the probability one, $p_{N-1, N}=1$, to the state $j=N-1$ ). Furthermore, from the state $j=1$ the channel can undergo a transition into its open state $j=0$ with the rate $\gamma$, or make transition into the manifold of conformational diffusion substates with the rate $\kappa$. For this state, the corresponding RTD reads $\psi_{1}(\tau)=(\gamma+\kappa) \exp [-(\gamma+\kappa) \tau]$ and the transition probabilities are $p_{01}=\gamma /(\gamma+\kappa)$ and $p_{21}=\kappa /(\gamma+\kappa)$. The dynamics of state occupancies $p_{j}(t)$ is described by the generalized master equation (GME) due to Kenkre, Montroll and Shlesinger [28, 29] and its generalization [30, 31]. The corresponding dynamics reads (with an initial preparation in some state $\left.j_{0}, p_{j_{0}}(0)=1\right)$ :

$$
\begin{aligned}
\dot{p}_{j}(t) & =\int_{0}^{t} K\left(t-t^{\prime}\right)\left[p_{j-1}\left(t^{\prime}\right)+p_{j+1}\left(t^{\prime}\right)-2 p_{j}\left(t^{\prime}\right)\right] d t^{\prime}, j=\overline{3, N-2} \\
\dot{p}_{N}(t) & =\int_{0}^{t} K\left(t-t^{\prime}\right) p_{N-1}\left(t^{\prime}\right) d t^{\prime}-\int_{0}^{t} K_{N}\left(t-t^{\prime}\right) p_{N}\left(t^{\prime}\right) d t^{\prime} \\
\dot{p}_{N-1}(t) & =\int_{0}^{t} K\left(t-t^{\prime}\right)\left[p_{N-2}\left(t^{\prime}\right)-2 p_{N-1}\left(t^{\prime}\right)\right] d t^{\prime}+\int_{0}^{t} K_{N}\left(t-t^{\prime}\right) p_{N}\left(t^{\prime}\right) d t^{\prime} \\
\dot{p}_{2}(t) & =\int_{0}^{t} K\left(t-t^{\prime}\right)\left[p_{3}\left(t^{\prime}\right)-2 p_{2}\left(t^{\prime}\right)\right] d t^{\prime}+\kappa p_{1}(t) \\
\dot{p}_{1}(t) & =\int_{0}^{t} K\left(t-t^{\prime}\right) p_{2}\left(t^{\prime}\right) d t^{\prime}-(\kappa+\gamma) p_{1}(t)+k_{c} p_{0}(t), \\
\dot{p}_{0}(t) & =-k_{c} p_{0}(t)+\gamma p_{1}(t),
\end{aligned}
$$

with the kernels $K(t)$ and $K_{N}(t)$ defined through their Laplace-transforms

$$
\begin{aligned}
\tilde{K}(s) & =\frac{1}{2} \frac{s \tilde{\psi}(s)}{1-\tilde{\psi}(s)}, \\
\tilde{K}_{N}(s) & =\frac{s \tilde{\psi}_{N}(s)}{1-\tilde{\psi}_{N}(s)},
\end{aligned}
$$

where $\tilde{\psi}(s)$ and $\tilde{\psi}_{N}(s)$ are the Laplace-transforms of $\psi(\tau)$ and $\psi_{N}(\tau)$, respectively. These kernels can be derived due to the following simple consideration. Let us prepare the diffusing particle at $t=0$ in the state $j=2 \ldots N-1$ and let it make transitions to the neighboring states without return. Then, the survival probability $\Phi(t)=\int_{t}^{\infty} \psi(\tau) d \tau$ in the state $j$ (which should be identified under such conditions with $p_{j}(t)$ ) is governed obviously by the following equation $\dot{\Phi}(t)=-2 \int_{0}^{t} K\left(t-t^{\prime}\right) \Phi\left(t^{\prime}\right) d t^{\prime}$ with $\Phi(0)=1$. It can be easily solved by the Laplace-transform method to yield the first equation in Eq. (9) by taking into account $\tilde{\psi}(s)=1-s \tilde{\Phi}(s)$. The second kernel $K_{N}(t)$ is obtained along the same lines.

The RTD of the open state is readily obtained, i.e. $\psi_{o}(\tau)=k_{c} \exp \left(-k_{c} \tau\right)$. In order to calculate the RTD of the set of closed states one starts out from $p_{1}(0)=1$ (the channel has been just closed) to obtain the survival probability $\Phi_{c}(t)=\sum_{j=1}^{N} p_{j}(t)$ with the boundary condition that the state $j=0$ is absorbing. This latter condition is realized by setting formally $k_{c} \rightarrow 0$. The corresponding RTD then follows from $\psi_{c}(\tau)=-d \Phi_{c}(\tau) / d \tau$. The total population of the closed state $p_{c}(t)=\sum_{j=1}^{N} p_{j}(t)$ obeys (not allowing for the backward transition, $k_{c} \rightarrow 0$ ):

$$
\dot{p}_{c}(t)=-\gamma p_{1}(t) \text {. }
$$


This must be used as the proper boundary condition (it yields a radiation boundary via continuity equation in the scaling limit, see below) to calculate $\psi_{c}(\tau)[33,34]$.

Next let us make the Ansatz that $\psi(\tau)=-\frac{d}{d \tau} E_{\alpha}\left(-(2 \kappa \tau)^{\alpha}\right)$, where $E_{\alpha}(z):=\sum_{0}^{\infty} z^{n} / \Gamma(\alpha n+1)$ is the Mittag-Leffler function. It is defined via a generalization of the Taylor series expansion of the $\operatorname{exponential~function,} E_{1}(z)=\exp (z)$ and $\Gamma(z)$ is the standard Gamma-function. In other words, the corresponding survival probability in the state just occupied [40], $\Phi(\tau)=\int_{\tau}^{\infty} \psi(t) d t$ is given by $\Phi(\tau)=E_{\alpha}\left(-(2 \kappa \tau)^{\alpha}\right)$. This corresponds to the Cole-Cole relaxation law, i.e. the relaxation law which yields the Cole-Cole susceptibility [35, 36]. The Laplace-transform of $\Phi(\tau)$ reads $\tilde{\Phi}(s)=s^{\alpha-1} /\left[s^{\alpha}+(2 \kappa)^{\alpha}\right][41,42]$ and by use of the relation, $\tilde{\psi}(s)=1-s \tilde{\Phi}(s)$, one obtains $\tilde{\psi}(s)=(2 \kappa)^{\alpha} /\left[s^{\alpha}+(2 \kappa)^{\alpha}\right]$. This particular choice of RTD interpolates between the initial stretched exponential (Weibull) distribution [40] (which corresponds to the Kohlrausch relaxation law; "stretched exponential" refers here to the survival probability $\Phi(\tau)$, with $\psi(\tau) \propto 1 / \tau^{1-\alpha}$ at $\tau \rightarrow 0$ ) and the asymptotic long time power law $\psi(\tau) \propto 1 / \tau^{1+\alpha}$. It yields anomalously slow diffusion, $\left\langle\delta x^{2}(t)\right\rangle \propto t^{\alpha}$ [42]. For example, such an anomalous diffusion is measured experimentally, along with the RTD in trapping domains exhibiting the corresponding power law, for colloidal particles in cytoskeleton actin networks of biological cells [37] and in living cells [38]. These latter experimental results offer a clear clue for understanding the results on virus diffusion in infected cells [39] - that is an observed anomalously slow diffusion in actin networks combined with active directional trafficking of viruses by molecular motor proteins 39]. For the discussed form of RTD, $\tilde{K}(s)=(2 \kappa)^{\alpha} s^{1-\alpha} / 2$ and the fractional master equation follows exactly from Eq. (3) as a particular case of the GME. It reads explicitly,

$$
\dot{p}_{j}(t)=\frac{1}{2}(2 \kappa)^{\alpha}{ }_{0} \hat{D}_{t}^{1-\alpha}\left[p_{j-1}\left(t^{\prime}\right)+p_{j+1}\left(t^{\prime}\right)-2 p_{j}\left(t^{\prime}\right)\right]
$$

where ${ }_{0} \hat{D}_{t}^{1-\alpha}(\ldots)=\frac{1}{\Gamma(\alpha)} \frac{\partial}{\partial t} \int_{0}^{t} \frac{(\ldots) d t^{\prime}}{\left(t-t^{\prime}\right)^{1-\alpha}}$ is the integro-differential operator of fractional derivative introduced by Riemann and Liouville, see Refs. 41, 42, 43, 46] for reviews and further references. In the case of a two-state dynamics a similar fractional master equation was obtained in [4, 45]. Note that the fractional master equation (11) presents in fact a conventional generalized master equation being nonlocal in time. The introduction of a fractional time derivative in the generalized master equation of CTRW is nothing but a shorthand notation which corresponds to a specific choice of the RTD. The importance of this equation lies in the fact that it can serve as a useful mathematical tool to model anomalously slow diffusion. The physical origin of this diffusion can be attributed to very broad residence time distributions on the sites of particle localization with diverging mean residence time [23, 25, 26]. In practice this implies that the corresponding mean residence time is exceedingly large as compared with the characteristic time scale of anomalous diffusion in the given domain of a finite size. As a consequence, the approximation with an infinite mean residence time becomes physically justified.

Likewise, with $\psi_{N}(\tau)=-\frac{d}{d \tau} E_{\alpha}\left(-(\kappa \tau)^{\alpha}\right)$, Eq. (4) takes on the form

$$
\dot{p}_{N}(t)=\frac{1}{2}(2 \kappa)^{\alpha}{ }_{0} \hat{D}_{t}^{1-\alpha} p_{N-1}\left(t^{\prime}\right)-\kappa^{\alpha}{ }_{0} \hat{D}_{t}^{1-\alpha} p_{N}\left(t^{\prime}\right)
$$

The remaining equations (5), (6), and (7) involving the memory kernel can readily be rewritten in a similar form upon use of the notation of the fractional time derivative.

It is worth noting that Eq. (11) and Eq. (12) can equivalently be brought into a form with the fractional Caputo derivative $D_{*}^{\alpha}(\ldots):=\frac{1}{\Gamma(1-\alpha)} \int_{0}^{t} \frac{\frac{\partial}{\partial t^{\prime}}(\ldots) d t^{\prime}}{\left(t-t^{\prime}\right)^{\alpha}}$ [41] in the left hand side. Namely, Eq. (11) becomes

$$
D_{*}^{\alpha} p_{j}\left(t^{\prime}\right)=\frac{1}{2}(2 \kappa)^{\alpha}\left[p_{j-1}(t)+p_{j+1}(t)-2 p_{j}(t)\right]
$$

Such a fractional master equation has been first derived from CTRW in Ref. [8]. This form is, however, clearly not appropriate for a further use in the studied case, where the normal rate transitions are also present. The reason is that the Eqs. (6), (7) would get a form mixing the fractional Caputo derivative and the fractional Riemann-Liouville integral in left and right hand sides of the corresponding equations. This fact establishes a clear advantage for the use of the Riemann-Liouville fractional derivative in a case where normal rate processes are also present.

\section{A. Scaling limit to a fractional diffusion equation}

Let us perform next a continuous limit: namely, assuming the distance $\Delta x$ between neighboring sites we introduce the conformational coordinate $x:=-j \Delta x$ which models the manifold of closed diffusion substates. The following continuous limit is assumed: Let $\Delta x \rightarrow 0, N \rightarrow \infty, \kappa \rightarrow \infty$ whereas keeping $K_{\alpha}:=\frac{1}{2}(2 \kappa)^{\alpha}(\Delta x)^{2}$ and the diffusion 
"length" $L:=N \Delta x$ constant. By use of the expansion: $p_{j \pm 1}(t) / \Delta x:=P(-[j \pm 1] \Delta x, t) \approx P(x, t) \mp \frac{\partial P(x, t)}{\partial x} \Delta x+$ $\frac{1}{2} \frac{\partial^{2} P(x, t)}{\partial x^{2}}(\Delta x)^{2}$, in (3) we arrive at the following fractional diffusion equation in continuous state space, i.e.,

$$
\frac{\partial P(x, t)}{\partial t}=K_{\alpha} \hat{D}_{t}^{1-\alpha} \frac{\partial^{2} P\left(x, t^{\prime}\right)}{\partial x^{2}}
$$

where $K_{\alpha}$ is the diffusion constant of anomalously slow diffusion. The fractional diffusion equation (14) presents a non-Markovian diffusion equation [27] with a particular choice of an integral kernel that accounts for the residence time distributions following the Cole-Cole relaxation law [35, 36] in a corresponding formulation in terms of a discrete CTRW. The fractional diffusion equation (14) assumes the form of a continuity equation,

$$
\frac{\partial P(x, t)}{\partial t}=-\frac{\partial J(x, t)}{\partial x}
$$

where the probability flux $J(x, t)$ becomes modified due to the fractional time-derivative,

$$
J(x, t)=-K_{\alpha 0} \hat{D}_{t}^{1-\alpha} \frac{\partial P\left(x, t^{\prime}\right)}{\partial x} .
$$

Our derivation of the fractional diffusion equation from the CTRW complements previous studies [42, 47]; it is rather simple and does not require jumps with a variable step length beyond nearest neighbors. For this very reason, no overflights of the boundaries occur that are possible otherwise. This observation is of crucial importance in determining the physically correct boundary conditions [49, 50]. This also means that the boundaries are strictly local in space. The given derivation removes any open query about the space-locality of boundary conditions for the fractional diffusion equation. After the integration of the continuity equation from $x=-L$ to $x=0$, one deduces that the decrease of the total probability of the closed state manifold (the survival probability), $p_{c}(t)=\int_{-L}^{0} P(x, t) d x$ occurs due to the probability flux on the boundary. Accordingly, we will replace the original discrete master equation in space by its corresponding fractional diffusion equation with the following boundary conditions as they emerge from the original problem. The boundary $x=-L$ is a reflecting one, obeying :

$$
J(-L, t)=0 .
$$

The boundary at $x=0$ is radiative. Setting $p_{1}(t) \approx \Delta x P(0, t)=L P(0, t) / N$ and using Eq. (10) one finds

$$
J(0, t)=\gamma L P(0, t) / N
$$

We additionally use here the specific scaling limit: $\gamma \rightarrow \infty, N \rightarrow \infty$ with $\left\langle\tau_{c}\right\rangle=N / \gamma$ being held fixed. This quantity possesses the meaning of the mean residence time in the closed state manifold, see below. The radiation boundary acquires then the explicit form

$$
J(0, t)=L P(0, t) /\left\langle\tau_{c}\right\rangle
$$

Our fractional diffusion modeling for the RTD of closed time intervals thus has three parameters only: (i) the mean residence time $\left\langle\tau_{c}\right\rangle$, (ii) the characteristic time of conformational diffusion, i.e. $\tau_{D}:=\left(L^{2} / K_{\alpha}\right)^{1 / \alpha}$ and (iii) the power law index of anomalous diffusion $\alpha$. In the case of normal diffusion, i.e. $\alpha=1$, the special scaling limit used here can also be justified from a Kramers approach [51] to the gating problem [11, 12]. Whereas the solution of the Kramers approach in Ref. [11] in addition yields also an analytical expression for $\left\langle\tau_{c}\right\rangle$, which reproduces the experimental crossover behavior from an exponential-to-linear voltage dependence due to Hodgkin and Huxley [52], the model here treats $\left\langle\tau_{c}\right\rangle$ as one of the phenomenological parameters. It is also worthwhile to remark that the boundary condition does not contain the index of anomalous diffusion $\alpha$. Formally it remains the same as for normal diffusion. The flux expression (16) is, however, not local in time. Moreover, the r.h.s. of (19) does not contain the fractional derivative in time. This feature is in accord with the original discrete model where the last, final transition into the open state is given by an ordinary rate transition. The analogy with the Kramers model of Ref. [11] is that the diffusion in the domain of voltage-dependent states (cf. 11, 12]), which becomes a thin boundary layer in the considered scaling limit, remains normal. This justifies well the use of boundary condition (19) in our fractional diffusion model which now is completely formulated.

\section{B. Characteristics of the residence time distribution}

To obtain the distribution of closed residence times $\psi_{c}(\tau)$ one needs to solve first the fractional diffusion equation (14) with the boundary conditions (17) and (19) and the initial condition $P(x, 0)=\delta\left(x-x_{0}\right)$ with $x_{0} \rightarrow 0_{-}$. 
The survival probability $\Phi_{c}(t)$ follows as the integral of the solution over the spatial variable and subsequently the corresponding RTD follows as the negative time derivative of the survival probability. This task has been achieved by use of the Laplace-transform method. The details of the derivation are outlined in the Appendix. The final result for the Laplace-transformed RTD of closed time intervals then reads:

$$
\tilde{\psi}_{c}(s)=\frac{1}{1+s\left\langle\tau_{c}\right\rangle g_{\alpha}\left(s \tau_{D}\right)},
$$

where an auxiliary function

$$
g_{\alpha}(z)=\frac{\tanh \left[z^{\alpha / 2}\right]}{z^{\alpha / 2}}
$$

has been introduced. For $\alpha=1$, this result reduces to one for normal diffusion in Ref. 11, 12]. Moreover, since $g_{\alpha}(z)=$ $1+o(z)$ for small $z$, one can readily see from Eq. (20) that $\left\langle\tau_{c}\right\rangle$ indeed has the meaning of a mean residence time, $\left\langle\tau_{c}\right\rangle:=\int_{0}^{\infty} \tau \psi_{c}(\tau) d \tau=-\left.\frac{d \tilde{\psi}(s)}{d s}\right|_{s=0}$. Note also that the second moment of RTD diverges, $\left\langle\tau_{c}^{2}\right\rangle:=\int_{0}^{\infty} \tau^{2} \psi_{c}(\tau) d \tau \rightarrow \infty$ for all $\alpha<1$ (anomalous diffusion). Furthermore, if $\tau_{D}=0$, then the closed time distribution becomes strictly exponential, i.e. $\psi_{c}(\tau)=\exp \left(-\tau /\left\langle\tau_{c}\right\rangle\right) /\left\langle\tau_{c}\right\rangle$ and the simplest two-state Markovian model of the ion channel gating is reproduced with the opening rate $k_{o}=\left\langle\tau_{c}\right\rangle^{-1}$. In general, the expression (20) cannot be inverted to the time domain exactly; its different characteristic regimes, however, can be discussed analytically.

In proceeding, let us consider first the limit of a large conformational diffusion time $\tau_{D} \gg\left\langle\tau_{c}\right\rangle$. Then (by use of the large- $z$ asymptotic behavior of $g(z) \sim z^{-\alpha / 2}$, we have

$$
\tilde{\psi}_{c}(s) \approx \frac{1}{1+\left(s \tau_{0}\right)^{1-\alpha / 2}}
$$

with $\tau_{0}:=\tau_{D}\left(\frac{\left\langle\tau_{c}\right\rangle}{\tau_{D}}\right)^{1 /(1-\alpha / 2)}$. The inversion of Eq. (22) is given as $\psi_{c}(\tau)=-d \Phi_{c}(\tau) / d \tau$ in terms of the survival probability

$$
\Phi_{c}(\tau)=E_{1-\alpha / 2}\left[-\left(\frac{\tau}{\tau_{0}}\right)^{1-\alpha / 2}\right],
$$

which is expressed through the Mittag-Leffler function $E_{\alpha}(z)$ and corresponds to the Cole-Cole relaxation law [35, 36]. Because $E_{1 / 2}\left(-z^{1 / 2}\right)=e^{z} \operatorname{erfc}\left(z^{1 / 2}\right)$ [42], where erfc $(z)$ is the complementary error function, the solution of the normal diffusion problem in Ref. [10] for the initial and intermediate time evolution regimes is reproduced from Eq. (23). For $\tau \ll \tau_{0}$, Eq. (23) behaves as a stretched exponential [42],

$$
\Phi_{c}(\tau) \approx \exp \left[-\frac{1}{\Gamma(2-\alpha / 2)}\left(\frac{\tau}{\tau_{0}}\right)^{1-\alpha / 2}\right] .
$$

This dependence (24) corresponds in the language of time-dependent rates $k_{o}(\tau):=-\frac{d}{d \tau} \ln \left[\Phi_{c}(\tau)\right]$ (used in the renewal theory) [6, 40, 53] to $k_{o}(\tau) \propto \tau^{-\alpha / 2}$. Such a time-dependent rate of recovery from inactivation has been measured with $\alpha=1$ for a sodium ion channel in Ref. [54]. In the limit $\tau \rightarrow 0$, Eq. (24) yields a power law for the RTD,

$$
\psi_{c}(\tau) \propto \tau^{-\alpha / 2} .
$$

Furthermore, for intermediate times $\tau_{0} \ll \tau \ll \tau_{D}$, Eq. (23) yields another power law, reading

$$
\psi_{c}(\tau) \propto \tau^{-\beta}
$$

with $\beta=2-\alpha / 2$. For $\alpha=1$ such an intermediate power law with the slope $\beta=3 / 2$ has been measured for a potassium ion channel in [8]. Note that this particular power law exponent reflects normal diffusion. Consequently, the origin of the intermediate power law is principally not due to the anomalous diffusion behavior. Our theory smoothly reproduces the intermediate power law associated with normal diffusion in the limit $\alpha \rightarrow 1$.

Other power law features were also measured in experiments, for example, for a gramicidin channel with the slope $\beta \approx 1.7$ [17]. This corresponds to an intermediate power law in Eq. (26) with $\alpha \approx 0.6$. Moreover, power law exponents with $\beta>2$ are also measured in experiments [18] analyzed in Ref. [19] from a pure phenomenological perspective without clarifying a tentative mechanism. Our model can as well capture such anomalous power laws which cannot be explained within the intermediate power law asymptotics (26). 
Indeed, let us assume that $\tau_{D}$ is sufficiently small and to consider the asymptotic behavior $\tau \rightarrow \infty$. The corresponding asymptotics can be deduced from the behavior of $\tilde{\psi}_{c}(s)$ at small $s$. For $s \rightarrow 0$, Eq. (20) yields,

$$
\tilde{\psi}_{c}(s) \approx 1-s\left\langle\tau_{c}\right\rangle\left[1-\left(s \tau_{D}\right)^{\alpha} / 3\right]
$$

From this, by way of $\tilde{\Phi}_{c}(s)=\left(1-\tilde{\psi}_{c}(s)\right) / s \approx\left\langle\tau_{c}\right\rangle\left(1-\left(s \tau_{D}\right)^{\alpha} / 3\right)$ for $s \rightarrow 0$, it follows 5.5 that $\Phi_{c}(\tau) \propto 1 / \tau^{1+\alpha}$ for $\tau \rightarrow \infty$. This renders then a power law (26) with $\beta=2+\alpha$ for large $\tau$. This asymptotic power law with $\beta>2$ is a manifestation of the anomalously slow conformational diffusion in a space domain of finite size. It replaces an exponential asymptotic behavior of $\psi_{c}(\tau)$ for $\tau>\tau_{D}$ in the case of normal diffusion [7, 11, 12].

\section{APPLICATION TO GATING DYNAMICS OF A LOCUST POTASSIUM ION CHANNEL}

Our fractional diffusion model can be used to describe the rather complex gating behavior observed for a locust potassium ion channel [19]. This ion channel exhibits experimentally a Pareto law in its gating kinetics, $\psi_{c}(\tau)=$ $a /(b+\tau)^{\beta}$ with $\beta \approx 2.24 \pm 0.06$. The corresponding autocorrelation function, however, seems to exhibit three different interchanging power laws [19]. These features are compatible with our model. Within a two-state reduction we are dealing with an alternating renewal process [4]. Its (Laplace-transformed) normalized autocorrelation function reads 56, 57]

$$
\tilde{k}(s)=\frac{1}{s}-\left(\frac{1}{\left\langle\tau_{o}\right\rangle}+\frac{1}{\left\langle\tau_{c}\right\rangle}\right) \frac{1}{s^{2}} \frac{\left(1-\tilde{\psi}_{o}(s)\right)\left(1-\tilde{\psi}_{c}(s)\right)}{\left(1-\tilde{\psi}_{o}(s) \tilde{\psi}_{c}(s)\right)} .
$$

For our case under study this yields

$$
\tilde{k}(s)=\frac{1}{s} \frac{f_{\alpha}\left(s \tau_{D}\right)+s\left\langle\tau_{c}\right\rangle}{1+\frac{\left\langle\tau_{c}\right\rangle}{\left\langle\tau_{o}\right\rangle}+f_{\alpha}\left(s \tau_{D}\right)+s\left\langle\tau_{c}\right\rangle},
$$

where $f_{\alpha}(z):=1 / g_{\alpha}(z)-1$. Note that $f_{\alpha}(0)=0$ and for $\tau_{D}=0$ the inversion of (29) yields the Markovian result $k(t)=\exp \left[-\left(k_{0}+k_{c}\right) t\right]$. Moreover, the analytical expression (29) allows one to study the asymptotics of $k(t)$ at $t \rightarrow \infty$. Namely, from $f_{\alpha}\left(s \tau_{D}\right) \approx\left(s \tau_{D}\right)^{\alpha} / 3$ it follows that $\tilde{k}(s) \propto s^{\alpha-1}$ at $s \rightarrow 0$. By virtue of a Tauberian theorem [58], this latter result readily yields,

$$
k(t) \propto t^{-\alpha}
$$

This power law feature agrees well with the experiment which shows asymptotically (30) with $\alpha=0.28 \pm 0.1$. Furthermore, an intermediate asymptotics of $k(\tau)$ can be obtained by studying the limit of very large $\tau_{D}$. Using the scaling $\tilde{s}:=s\left\langle\tau_{c}\right\rangle$ and the limit of very large values $y:=\tau_{D} /\left\langle\tau_{c}\right\rangle \gg 1$ such that $\tilde{s} \ll 1$ is allowed for, whereas still $\tilde{s} y \gg 1$, Eq. 29) can formally be approximated by

$$
\tilde{k}(s)=\frac{s^{\alpha / 2-1}}{s^{\alpha / 2}+r^{\alpha / 2}},
$$

with $r=\tau_{D}^{-1}\left(1+\left\langle\tau_{c}\right\rangle /\left\langle\tau_{o}\right\rangle\right)^{2 / \alpha}$. The formal inversion of Eq. (31) yields $k(t)=E_{\alpha / 2}\left[-(r t)^{\alpha / 2}\right]$. This in turn yields an intermediate asymptotics $k(\tau) \propto \tau^{-\alpha / 2}$ within $\left\langle\tau_{c}\right\rangle \ll \tau \ll \tau_{D}$. Indeed, the analysis of experimental data in [19] reveals such an intermediate asymptotics $k(\tau) \propto \tau^{-0.14 \pm 0.02}$. The numerical inversion of $\tilde{k}(s)$ in Fig. 2 displays three different power law regimes in qualitative agreement with the experimental data. Only one of these power laws - the long-time asymptotical one - seems, however, to present a true power law asymptotics. The intermediate power law in Fig. 2 does not agree numerically with the experimental one in Ref. [19]. Nevertheless, the experimental data agree - surprisingly enough - with the intermediate asymptotics obtained above in the limit $\tau_{D} \rightarrow \infty$.

Furthermore, the numerical inversion of $\tilde{\psi}_{c}(s)$ in Fig. 3 can be fitted by Pareto law with $\beta \approx 2.24$. The discrepancy between $\beta-2 \approx 0.24$ and $\alpha=0.28$ is due to the experimental restrictions on the maximal time intervals measured. The actual power law asymptotics for $\tau \rightarrow \infty$ in Fig. 3 is $\Phi_{c}(\tau) \propto \tau^{-1.28}$. This long time asymptotic regime is not yet attained in Fig. 3 which instead closely agrees with $\Phi_{c}(\tau) \propto \tau^{-1.24}$, see in Fig. 3, giving an apparent agreement with the experimental data. In view of our few elementary model assumptions, the agreement between theory and the experimental data [18] analyzed in Ref. [19] is striking indeed.

Our fractional diffusion scheme is not expected to describe the experimental facts quantitatively in all details. In particular, it predicts that the low-frequency part of the spectral power $S(f)$ of ion current fluctuations of the locust 


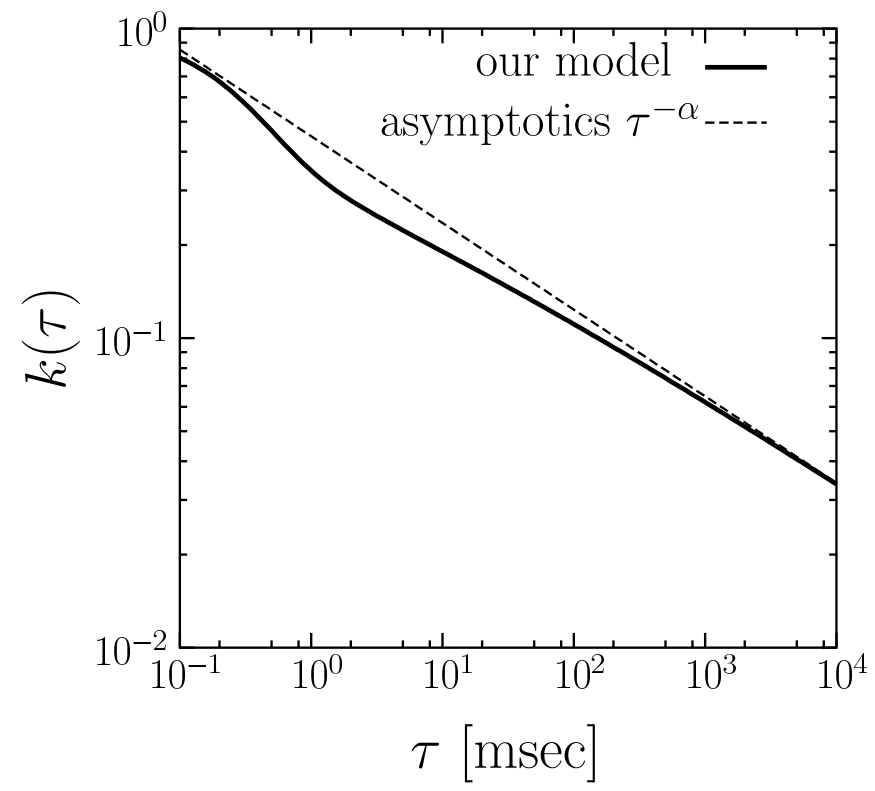

FIG. 2: Normalized autocorrelation function of conductance fluctuations. Numerical inversion of Eq. (29) is done with the (improved) Stehfest algorithm [60] for the following set of parameters: $\left\langle\tau_{c}\right\rangle=0.84$ msec, $\left\langle\tau_{o}\right\rangle=0.79$ msec [19] and assumed $\tau_{D}=100 \mathrm{msec}$ and $\alpha=0.28$.

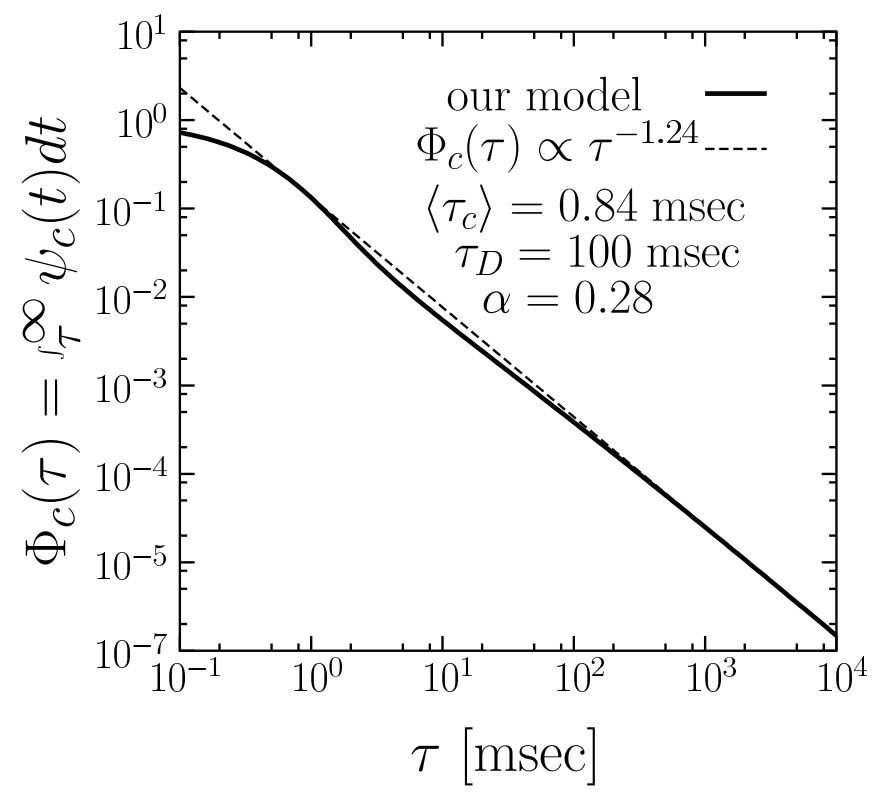

FIG. 3: Survival probability of the closed state for the studied model. The set of parameters is the same as in Fig.2.

BK potassium ion channel corresponds to $1 / f^{\gamma}$ noise with $\gamma=1-\alpha \approx 0.72$ [57, 59. The experiment 61] indeed reveals $1 / f^{\gamma}$ noise with $\gamma$ close but to unity, $\gamma \approx 1$. The reason for this discrepancy is not resolved. The asymptotic behavior of the autocorrelation function in Ref. [19] and the behavior of the low-frequency part of the spectrum in Ref. 61] are certainly at odds. A possible reason could be nonstationarity of the given current recordings. Nevertheless, the qualitative agreement, i.e., the principal theoretical prediction and the measurement of $1 / f^{\gamma}$ noise, is comforting.

Moreover, the durations of residence time intervals in open and closed states can be correlated. Such correlations can be induced by stochastic binding of calcium ions which regulate the gating dynamics of large conductance potassium ion channels. To account for such correlation effects, our model principally can be generalized in the same spirit like the original diffusion model has been generalized to include ligand binding effects [2]. This generalization is left but 
for a future study.

\section{DISCUSSION AND CONCLUSION}

The gating dynamics of protein ion channels in biological membranes is governed by a conformational dynamics on a very complex energy landscape with a huge number degrees of freedom. This multidimensional energy landscape can possess deep energy wells (as compared with the thermal energy $k_{B} T$ ) which are separated by potential barriers. In addition, there can exist an underlying energy valley network connecting these wells which results in an energy quasidegeneracy. The traditional discrete state approach to the gating dynamics pursued by the community of molecular physiologists presents an abstraction to this complexity: it has its focus on the fact of deep potential wells being separated by high energy barriers. The energy quasi-degeneracy of potential wells enters the theory as an entropic contribution to the corresponding free energies after reduction of the multidimensional reality to low-dimensional models (possessing a few discrete states only). This traditional approach has proven useful over the past years and it serves as a serviceable working tool for the analysis of the experimental data. This approach is, however, not able to capture the physical origin of such complexity features as the presence of power law distributions of the residence time intervals, the slow decay of the autocorrelations of fluctuations or the presence of $1 / f^{\gamma}$ noise feature in the power spectrum of fluctuations of several ion channel types, to name but a few. Experiments have demonstrated [63] that the $1 / f^{\gamma}$ noise is due to the conformational transitions among different conductance states. In particular, the ion current is free of $1 / f^{\gamma}$ noise in a frozen conductance substate of the ion channel. Therefore, the $1 / f^{\gamma}$ noise originates due to fluctuations among experimentally distinguishable substates [63]. These features reveal unambiguously the non-Markovian character of the observed "on-off" ion current fluctuations 6, 64, 65]. The diffusion models of ion channel gating 1, 9, 10, 11, 12, 13, 14, 15] present another, complementary abstraction of the actual dynamics. These approaches attempt to capture the spatial structures of the potential minima and the associated dynamics, and/or the corrugated and hierarchical features of the real multidimensional conformation landscape after performing the reduction to a reaction coordinate picture [20]. It is physically likely that the ion channel protein can become temporarily trapped in some domains of its intrinsic conformational landscape from which it can escape by activated jumps among those states. Due to a complicated structure of such traps the corresponding residence time distribution can possess a divergent, - or from a practical point of view - a very large first moment. This in turn gives rise to an anomalous conformation diffusion within the chosen reduced reaction coordinate description. Our scheme in terms of a fractional, continuous diffusion model, being complemented with appropriate boundary conditions properly accounts for such complexity.

As demonstrated theoretically and exemplified with the gating of a BK locust potassium ion channel our fractional diffusion theory presents a powerful approach to describe these various observed power law characteristics of the underlying gating dynamics.

\section{Acknowledgments}

This work has been supported by the Deutsche Forschungsgemeinschaft via the collaborative research centre, $M a-$ nipulation of matter on the nanoscale, SFB-486, project A-10.

\section{APPENDIX A: SOLUTION OF THE BOUNDARY-VALUE PROBLEM}

The Laplace-transformed probability $\tilde{P}(x, s):=\int_{0}^{\infty} e^{-s t} P(x, t) d t$, Eq. (14) reads:

$$
s \tilde{P}(x, s)-\delta\left(x-x_{0}\right)=K_{\alpha} s^{1-\alpha} \frac{d^{2} \tilde{P}(x, s)}{d x^{2}}
$$

with $-L<x_{0}<0$. Note that the limit $x_{0} \rightarrow 0_{-}$will be taken at the very end of calculation. The corresponding Laplace-transformed boundary conditions assume the form

$$
\begin{aligned}
\left.\frac{d \tilde{P}(x, s)}{d x}\right|_{x=-L} & =0, \\
\left.s^{1-\alpha} \frac{d \tilde{P}(x, s)}{d x}\right|_{x=0} & =-\frac{L}{K_{\alpha}\left\langle\tau_{c}\right\rangle} \tilde{P}(0, s) .
\end{aligned}
$$


The challenge is thus the solution of the boundary-value problem (A1)-A3). Towards this goal we consider separately the solution in the domains, $-L<x<x_{0}$,

$$
\tilde{P}_{1}(x, s)=A_{1} \exp \left(\sqrt{\frac{s^{\alpha}}{K_{\alpha}}} x\right)+B_{1} \exp \left(-\sqrt{\frac{s^{\alpha}}{K_{\alpha}}} x\right),
$$

and $x_{0}<x<0$,

$$
\tilde{P}_{2}(x, s)=A_{2} \exp \left(\sqrt{\frac{s^{\alpha}}{K_{\alpha}}} x\right)+B_{2} \exp \left(-\sqrt{\frac{s^{\alpha}}{K_{\alpha}}} x\right) .
$$

At $x=x_{0}$, the solution is continuous,

$$
\tilde{P}_{1}\left(x_{0}, s\right)=\tilde{P}_{2}\left(x_{0}, s\right)
$$

The first derivative $d \tilde{P}(x, s) / d x$, however, experiences a jump. This can readily be seen upon integrating Eq. A1 in an infinitesimally small neighborhood of $x=x_{0}$. Thus,

$$
K_{\alpha} s^{1-\alpha}\left[\frac{d \tilde{P}_{2}(x, s)}{d x}-\frac{d \tilde{P}_{1}(x, s)}{d x}\right]_{x=x_{0}}=-1 .
$$

The coefficients $A_{1,2}$ and $B_{1,2}$ are determined by substitution of (A4) and A5 into Eqs. A2 , A3 A6 and (A7). Thereby, the proposed objective is exactly solved. The integration of the solution (A4) from $x=-L$ to $x=0\left(x_{0} \rightarrow 0_{-}\right)$yields the Laplace-transformed survival probability $\tilde{\Phi}_{c}(s)$; the corresponding RTD (20) follows as $\tilde{\psi}_{c}(s)=1-s \tilde{\Phi}_{c}(s)$.

[1] B. Hille, Ionic Channels of Excitable Membranes, 3d ed. (Sinauer Associates, Sunderland, 2001).

[2] Single-Channel Recording, 2nd ed., edited by B. Sakmann and N. Neher (Plenum, New York, 1995).

[3] D. Colquhoun, A. G. Hawkes, Phil. Trans. R. Soc. Lond. B 300, 1 (1982).

[4] F. Bezanilla, E. Perozo, E. Stefani, Biophys. J. 66, 1011 (1994).

[5] B. M. Rodriguez, D. Sigg, F. Bezanilla, J. Gen. Physiol. 112, 223 (1998).

[6] L. S. Liebovitch and J. M. Sullivan, Biophys. J. 52, 979 (1987); L. S. Liebovitch, J. Fishbarg, J. P. Koniarek, Math. Biosci. 84, 37 (1987).

[7] G. L. Millhauser, E. E. Salpeter, R. E. Oswald, Proc. Natl. Acad. Sci. USA 85, 1503 (1988).

[8] M. S. P. Sansom, F. G. Ball, C. J. Kerry, R. McGee, R. L. Ramsey, P. N. R. Usherwood, Biophys. J. 56, 1229 (1989).

[9] C. A. Condat, J. Jäckle, Biophys. J. 55, 915 (1989).

[10] W. Nadler and D. L. Stein, Proc. Natl. Acad. Sci. USA 88, 6750 (1991).

[11] I. Goychuk and P. Hänggi, Proc. Natl. Acad. Sci. USA 99, 3552 (2002).

[12] I. Goychuk and P. Hänggi, Physica A 325, 9 (2003).

[13] P. Läuger, Biophys. J. 53, 877 (1988).

[14] D. G. Levitt, Biophys. J. 55, 489 (1989).

[15] R. Shirokov, G. Ferreira, J. Yi, and E. Rios, J. Gen. Physiol. 111, 807 (1998).

[16] D. Sigg, H. Qian, and F. Bezanilla, Biophys. J. 76, 782 (1999).

[17] A. Ring, Biochim. Biophys. Acta 856, 646 (1986).

[18] E. Gorczynska, P. L. Huddie, B. A. Miller, I. R. Mellor, H. Vais, R. K. Ramsey, and P. N. R. Usherwood, Pflügers Arch. Eur. J. Phys. 432, 597 (1996).

[19] S. Mercik and K. Weron, Phys. Rev. E 63, 051910 (2001).

[20] H. Frauenfelder, S. G. Sligar, and P. G. Wolynes, Science 254, 1598 (1991).

[21] H. Frauenfelder and B. H. McMahon, Ann. Phys. (Leipzig) 9, 655 (2000).

[22] F. G. Parak and G. U. Nienhaus, Chem. Phys. Chem. 3, 249 (2002).

[23] E. W. Montroll and G. H. Weiss, J. Math. Phys. 6, 167 (1965).

[24] M. Lax and H. Scher, Phys. Rev. Lett. 39, 781 (1977).

[25] B. D. Hughes, Random Walks and Random Environments, Volume 1: Random Walks (Clarendon Press, Oxford, 1995).

[26] M. F. Shlesinger, Random Processes, in: Encyclopedia of Applied Physics 16 (VCH, New York, 1996), pp. 45-70.

[27] G. H. Weiss, Aspects and Applications of the Random Walk (North-Holland, Amsterdam, 1994).

[28] V. M. Kenkre, E. W. Montroll and M. F. Shlesinger, J. Stat. Phys. 9, 45 (1973).

[29] G. H. Weiss and R. J. Rubin, Adv. Chem. Phys. 52, 363 (1983).

[30] A. I. Burshtein, A. A. Zharikov, S.I. Temkin, Theor. Math. Phys. 66, 166 (1986).

[31] See also [32] where the GME of Ref. [28] and its generalization by Burshtein, Zharikov and Temkin [30] (used here) are reproduced from the most general GME of CTRW with uncorrelated jumps, see Eq. (A8) in [32]. 
[32] I. Goychuk, Phys. Rev. E 70, 016109 (2004).

[33] N. G. van Kampen, Stochastic Processes in Physics and Chemistry, revised and enlarged edition (North-Holland, Amsterdam, 1992); P. Hänggi and H. Thomas, Phys. Rep.88, 207 (1982).

[34] N. W. Goel and N. Richter-Dyn, Stochastic Models in Biology (Academic Press, New York, 1974).

[35] K. Cole and R. Cole, J. Chem. Phys. 9, 341 (1941).

[36] R. Hilfer, J. Non-Cryst. Sol. 305, 122 (2002).

[37] I. Y. Wong, M. L. Gardel, D. R. Reichman, E. R. Weeks, M. T. Valentine, A. R. Bausch, and D. A. Weitz, Phys. Rev. Lett. 92, 178101 (2004).

[38] I. M. Tolić-Nørrelykke, E.-L. Munteanu, G. Thon, L. Oddershede, and K. Berg-Sørensen, Phys. Rev. Lett. 93, 078102 (2004).

[39] G. Seisenberger, M. U. Ried, T. Endreß, H. Büning, M. Hallek, C. Bräuchle, Science 294, 1929 (2001); C. Bräuchle, G. Seisenberger, Thomas Endreß, M.U. Ried, H. Büning, and M. Hallek, Chem.Phys.Chem. 3, 299 (2002).

[40] D. R. Cox, Renewal Theory, (Methuen, London, 1962).

[41] R. Gorenflo and F. Mainardi, in: Fractals and Fractional Calculus in Continuum Mechanics, edited by A. Carpinteri and F. Mainardi (Springer, Wien, 1997), pp. 223-276.

[42] R. Metzler and J. Klafter, Phys. Rep. 339, 1 (2000).

[43] I. M. Sokolov, J. Klafter and A. Blumen, Physics Today 55 (11), 48 (2002).

[44] I. M. Sokolov and R. Metzler, Phys. Rev. E 67, 010101 (2003).

[45] A. A. Stanislavsky, Phys. Rev. E 67, 021111 (2003).

[46] Fractional Calculus in Physics, edited by R. Hilfer (World Scientific, Singapore, 2000).

[47] R. Metzler, E. Barkai, and J. Klafter, Europhys. Lett. 46, 431 (1999); E. Barkai, R. Metzler, and J. Klafter, Phys. Rev. E 61, $132(2000)$.

[48] R. Hilfer and L. Anton, Phys. Rev. E 51, R848 (1995).

[49] C. Van den Broeck and P. Hänggi, Phys. Rev. A 30, 2730 (1984).

[50] V. Balakrishnan, C. Van den Broeck, and P. Hänggi, Phys. Rev. A 38, 4213 (1988).

[51] P. Hänggi, P. Talkner, and M. Borkovec, Rev. Mod. Phys. 62, 251 (1990); P. Hänggi, J. Stat. Phys. 42, 105 (1986); P. Hänggi, J. Stat. Phys. 44, 1003 (1986); (Addendum and Erratum).

[52] A. L. Hodgkin, A. F. Huxley, J. Physiol. (London) 117, 500 (1952).

[53] N. G. van Kampen, Physica A 96, 435 (1979).

[54] A. Toib, V. Lyakhov, and S. Marom, J. Neurosci. 18, 1893 (1998).

[55] M. A. Lavrentiev and B. V. Shabat, The Methods of Theory of Functions of Complex Variable, 5th ed. (Nauka, Moscow, 1987) (in Russian), theorem on p. 491.

[56] R. L. Stratonovich, Topics in the Theory of Random Noise (Gordon and Breach, NewYork, 1963), Vol. 1, p. 176.

[57] I. Goychuk and P. Hänggi, Phys. Rev. Lett. 91, 070601 (2003); I. Goychuk and P. Hänggi, Phys. Rev. E 69, 021104 (2004).

[58] G. Doetsch, Theorie und Anwendungen der Laplace-Transformation (Dover, New York, 1943).

[59] S. B. Lowen and M. C. Teich, Phys. Rev. E 47, 992 (1993).

[60] H. Stehfest, Comm. ACM 13, 47 (1970); Comm. ACM 13, 624 (1970); P.P. Valko and S. Vajda, Inverse Problems in Engineering 10, 467 (2002).

[61] Z. Siwy and A. Fulinski, Phys. Rev. Lett. 89, 158101 (2002).

[62] R. E. Oswald, G. L. Millhauser, and A. A. Carter, Biophys. J. 59, 1136 (1991).

[63] S. M. Bezrukov and M. Winterhalter, Phys. Rev. Lett. 85, 202 (2000); S. M. Bezrukov, Fluct. Noise Lett. 4, L23 (2004).

[64] B. J. West and W. Deering, Phys. Rep. 246, 1 (1994).

[65] A. Fulinski, Z. Grzywna, I. Mellor, Z. Siwy, and P. N. R. Usherwood, Phys. Rev. E 58, 919 (1998). 